\title{
EFFECT OF EXPERIENCE AND PSYCHOPHYSIOLOGICAL MODIFICATION BY COMBAT STRESS IN SOLDIER'S MEMORY
}

Delgado-Moreno, Rosa, Robles-Pérez, José Juan, Aznar-Laín, Susana, ClementeSuárez, Vicente Javier

\begin{abstract}
The present research aimed to analyze the effect of experience and psychophysiological modification by combat stress in soldier's memory in a simulated combat situation. Variables of rate of perceived exertion, blood glucose, blood lactate, lower body muscular strength manifestation, cortical arousal, specific fine motor skills, autonomic modulation, state anxiety, and memory and attention through a postmission questionnaire were analyzed before and after a combat simulation in 15 experienced soldiers of a special operation unit and 20 non-experienced soldiers of light infantry unit from the Spanish Army. The stress of combat simulation produces a significant increase $(p<0.05)$ in rated perceived exertion, blood glucose, blood lactate, somatic anxiety and a low frequency domain of the heart rate, and a significant decrease of rifle magazine reload time, high frequency domain of the heart rate and somatic anxiety in both groups. The variables of RPE, glucose, CFFT, RMRT, RMSSD, LF/HF, CA, SA and STAI were significantly different in experienced soldiers shown the activation of fight-flight system. The anticipatory anxiety in experienced soldiers shows a cognitive behavioral association by past experiences. The analysis of correct response in the postmission questionnaire show elements more related with the sight and that endanger the physical integrity of soldiers are more remembered, and some significant differences $(p<0.05)$ in the memory performance of experienced soldiers and non experienced soldiers where experienced soldiers shown a better performance. As conclusion, combat stress produce an increase in the psichophysiological response of soldiers independently of experience, but experienced ones presented a lower negative effect on memory than non experienced.
\end{abstract}

\section{Keywords}

Cognitive behavioral association, Combat stress, Memory, Military, Military anxiety 